\title{
An affine analogue of the Hartman-Nirenberg cylinder theorem
}

\author{
Maks A. Akivis and Vladislav V. Goldberg
}

\begin{abstract}
Let $X$ be a smooth, complete, connected submanifold of dimension $n<N$ in a complex affine space $A^{N}(\mathbb{C})$, and $r$ is the rank of its Gauss map $\gamma, \gamma(x)=T_{x}(X)$. The authors prove that if $2 \leq r \leq n-1, N-n \geq 2$, and in the pencil of the second fundamental forms of $X$, there are two forms defining a regular pencil all eigenvalues of which are distinct, then the submanifold $X$ is a cylinder with $(n-r)$-dimensional plane generators erected over a smooth, complete, connected submanifold $Y$ of rank $r$ and dimension $r$. This result is an affine analogue of the Hartman-Nirenberg cylinder theorem proved for $X \subset R^{n+1}$ and $r=1$. For $n \geq 4$ and $r=n-1$, there exist complete connected submanifolds $X \subset A^{N}(\mathbb{C})$ that are not cylinders.
\end{abstract}

Mathematics Subject Classification (2000): Primary 53A15. Secondary 53A05, 53A20.

Keywords and phrases: Developable surface, cylinder, Hartman-Nirenberg cylinder theorem, submanifold, degenerate Gauss mapping.

1. The Hartman-Nirenberg cylinder theorem. The Hartman-Nirenberg cylinder theorem in an $(n+1)$-dimensional Euclidean space $R^{n+1}$ was first proved in $[7]$. This theorem states:

Let $S: M \rightarrow R^{n+1}$ be a connected, $C^{2}$, orientable hypersurface in an $(n+1)$ dimensional space $R^{n+1}$. If $S$ is of constant zero curvature, then it is an $(n-1)$ cylinder (i.e., an n-dimensional cylinder with $(n-1)$-dimensional generators erected over a curve) in the sense that $S$ has a parametrization (in the large) of the form

$$
\vec{v}=\vec{v}(x)=\sum_{i=1}^{n-1} \vec{a}_{j} x^{j}+\vec{b}\left(x^{n}\right) \text { for all } x=\left(x^{1}, \ldots, x^{n}\right),
$$

where $\vec{a}_{1}, \ldots, \vec{a}_{n-1}$ are constant vectors in $R^{n+1}, \vec{b}\left(x^{n}\right)$ is a vector-valued function of a variable $x^{n}$ of class $C^{2}$ in $R^{n+1}$, and $\vec{a}_{1}, \ldots, \vec{a}_{n-1}, \partial \vec{b} / \partial x^{n}$ is a set of orthonormal vectors.

In the proof of this theorem, the authors first proved that the vanishing of the Gaussian curvature implies that the rank $r(x)$ of the Gaussian map of $S$ does not exceed one, $r(x) \leq 1$. If $r(x)=0$, then $S$ is a hyperplane. In the case $r(x)=1, S$ is an $(n-1)$-cylinder which can be parametrized as indicated in equation (1).

The proof of this theorem in [7] is based on the lemma on the constancy of a certain unique $(n-1)$-plane from the paper [6] of Chern and Lashof. Sternberg 19 called this lemma Lemma of Chern-Lashof-Hartman-Nirenberg. A projective analogue of this lemma is in [2], Theorem 4.1 (see also [3], Theorem 1).

Note that in [7] and [19], the authors obtain an $(n-1)$-cylinder, i.e., a cylinder in $R^{n+1}$ with $(n-1)$-dimensional plane generators erected over a curve. The reason that they did not get an $(n-r)$-cylinder, i.e., an $n$-dimensional cylinder in $R^{n+1}$ with $(n-r)$-dimensional plane generators erected over an $r$-dimensional submanifold, where $r=1, \ldots, n-1$, is that their theorem hypotheses imply that the rank $r(x)$ of the Gaussian map of $S$ does nor exceed one.

The authors noted in [7]: "A similar result under weaker differentiability hypotheses has been stated for $n=2$ by Pogorelov" (see [16] and [17]). For $R^{3}$, the results of [7] were developed further by Stocker (see [20] and [21). 
In recent papers (see for example, [8] and [9]) the authors state the HartmanNirenberg cylinder theorem by saying that "a properly embedded developable hypersurface in $R^{n+1}$ of rank $(\gamma) \leq 1$ is necessarily a cylinder". A similar result is known for a complex Euclidean space $C^{n+1}$ (see [1]; see also the survey [5]).

The authors would like to thank Professor Go-o Ishikawa for very useful discussions in the process of writing this paper. We also would like to thank J. Piontkowski who indicated an inaccuracy in the first version of our paper.

2. An affine analogue of the Hartman-Nirenberg cylinder theorem. The Hartman-Nirenberg cylinder theorem is of affine nature. In fact, the notion of a cylinder appearing in the theorem conclusion is an affine notion. As to the theorem hypotheses, although the notion of the Gaussian curvature is not affine, the notion of the rank $r$ of the Gauss map, which is fundamental in the proof of this theorem and whose boundedness, $r(x) \leq 1$, is implied by the vanishing of the Gaussian curvature, is even of projective nature. This is a reason that it is interesting to consider an affine analogue of the Hartman-Nirenberg cylinder theorem.

We recall that an $l$-cylinder $X$ in an affine space $A^{N}$ over the field of complex or real numbers is defined as a smooth $n$-dimensional submanifold bearing $l$-dimensional plane generators, $l<n$, which are parallel one to another. An $l$ cylinder is a tangentially degenerate submanifold of rank $r=n-l$. In an affine space $A^{N}, N>n$, an $l$-cylinder can be defined by a parametric equation

$$
\vec{\phi}\left(y^{1}, \ldots, y^{n}\right)=\sum_{j=1}^{l} \vec{a}_{j} y^{j}+\vec{b}\left(y^{l+1}, \ldots, y^{n}\right),
$$

where $\vec{a}_{j}$ are constant vectors in $A^{N}$ and $\vec{b}\left(y^{l+1}, \ldots, y^{n}\right)$ is a vector-valued function of $r=n-l$ variables defining in $A^{N}$ a director variety $Y$ of the cylinder $X$, and the vectors $\vec{a}_{j}$ and $\vec{b}_{p}=\frac{\partial \vec{b}}{\partial y^{p}}$ are linearly independent.

The aim of this paper is to prove the following affine analogue of the HartmanNirenberg cylinder theorem.

Theorem 1. Let $X^{n}$ be a smooth, complete, connected submanifold of constant rank $r, 2 \leq r \leq n-1$, in a complex affine space $A^{N}(\mathbb{C}), N-n \geq 2$. Suppose that in the pencil of the second fundamental forms of $X$, there are two forms defining a regular pencil all eigenvalues of which are distinct. Then the submanifold $X$ is a cylinder with $l$-dimensional plane generators, $l=n-r \geq 2$, and an $r$-dimensional tangentially nondegenerate director variety $Y$. In $A^{N}(\mathbb{C})$ such a cylinder can be defined by parametric equation (2).

We recall that the eigenvalues of a pencil of quadratic forms defined by the forms

$$
\phi^{\prime}=b_{p q}^{\prime} \theta^{p} \theta^{q} \text { and } \phi^{\prime \prime}=b_{p q}^{\prime \prime} \theta^{p} \theta^{q}
$$

are determined by the characteristic equation

$$
\operatorname{det}\left(b_{p q}^{\prime \prime}+\lambda b_{p q}^{\prime}\right)=0 .
$$

Note also that if a submanifold $X \subset A^{N}(\mathbb{C})$ is smooth (i.e., differentiable), then it is complex-analytic, and in particular, it is twice differentiable.

Note that the condition $N-n \geq 2$ in our cylinder theorem is essential since if $N-n=1$, in the space $A^{n+1}(\mathbb{C})$, there exist complete, connected, complex-analytic hypersurfaces $X^{n}$ of rank $r<n$ without singularities that are not cylinders. An example of such hypersurface $X^{3}$ in the space $A^{4}(\mathbb{C})$ was considered by Sacksteder 
in [18. An algebraic ruled noncylindrical hypersurface without singularities in $A^{4}(\mathbb{C})$ was constructed by Bourgain and published by $\mathrm{Wu}$ [22] (see also [8] and [9]). This example was studied in detail in 何.

Note that an affine cylinder theorem in other formulations was presented in the paper 10 by Nomizu and Pinkall (see also the book 11]) and in the papers [12], [13, and 14] by Opozda. Their affine cylinder theorems give sufficient conditions for a hypersurface (i.e., a submanifold of codimension one) $X$ in $A^{n+1}$ to be a cylinder erected over a curve with $(n-1)$-dimensional plane generators. Our affine cylinder theorem gives sufficient conditions for a submanifold $X$ of any codimension and any rank $r, 2 \leq r \leq n-1$, in $A^{N}, N-n \geq 2$, to be a cylinder erected over a submanifold of dimension $r$ and rank $r$ with $(n-r)$-dimensional plane generators. In a recent paper 15], Piontkowski considered in $P^{N}$ complete varieties with degenerate Gauss maps with rank equal 2 or 3 or 4 and with all singularities located at a hyperplane at infinity. In particular, as an extreme case, he obtained an affine cylinder theorem for varieties of rank 1 and any codimension. So, our affine cylinder theorem for submanifolds of codimension greater than 2 and rank $r \geq 2$ complements substantially all previously known affine cylinder theorems which were for hypersurfaces of rank 1 .

Theorem 1 is a simple consequence of a certain structure theorem (Lemma 2) which is of projective nature (see [3], where one can also find other structure theorems for submanifolds with degenerate Gauss map). In order to present this theorem, we need to introduce some notions and notations.

3. Basic equations and focal images. An $n$-dimensional submanifold $X$ of a projective space $P^{N}(\mathbb{C})$ is called tangentially degenerate if the rank of its Gauss mapping $\gamma: X \rightarrow G(n, N)$ is less than $n, r=$ rank $\gamma<n$. Here $x \in X, \gamma(x)=$ $T_{x}(X)$, and $T_{x}(X)$ is the tangent subspace to $X$ at $x$ considered as an $n$-dimensional projective space $P^{n}(\mathbb{C})$. The number $r$ is also called the $\operatorname{rank}$ of $X, r=\operatorname{rank} X$. We assume that the rank $r$ is constant on the submanifold $X$.

Denote by $L$ a leaf of this map, $L=\gamma^{-1}\left(T_{x}\right) \subset X$. It is easy to prove that a leave $L$ of the Gauss map $\gamma$ is a subspace of $P^{N}(\mathbb{C}), \operatorname{dim} L=n-r=l$ or its open part (see for example, [2], p. 115, Theorem 4.1).

The foliation on $X$ with leaves $L$ is called the Monge-Ampère foliation. As we did in [3], in this paper we extend the leaves of the Monge-Ampère foliation to a projective space $P^{l}(\mathbb{C})$ assuming that $L \sim P^{l}(\mathbb{C})$ is a plane generator of the submanifold $X$. In this sense the submanifold $X$ is complete. As a result, we have $X=f\left(P^{l}(\mathbb{C}) \times M^{r}\right)$, where $M^{r}$ is a complex variety of parameters, and $f$ is a differentiable map $f: P^{l}(\mathbb{C}) \times M^{r} \rightarrow P^{N}(\mathbb{C})$. The Monge-Ampère foliation is locally trivial but, as we will see later, its leaves $L$ can have singularities.

In $P^{N}(\mathbb{C})$, we consider a manifold of projective frames $\left\{A_{0}, A_{1}, \ldots, A_{N}\right\}$. On this manifold

$$
d A_{u}=\omega_{u}^{v} A_{v}, \quad u, v,=0,1, \ldots, N,
$$

where the sum $\omega_{u}^{u}=0$. The 1 -forms $\omega_{u}^{v}$ are linearly expressed in terms of the differentials of parameters of the group of projective transformations of the space $P^{N}(\mathbb{C})$. These 1 -forms satisfy the structure equations

$$
d \omega_{u}^{v}=\omega_{u}^{w} \wedge \omega_{w}^{v}
$$

of the space $P^{N}(\mathbb{C})$ (see, for example, [2], p. 19). Equations (4) are the conditions of complete integrability of equations $(3)$. 
Consider a tangentially degenerate submanifold $X \subset P^{N}(\mathbb{C}), \operatorname{dim} X=n$, rank $X=$ $r<n$. In addition, as above, let $L$ be a plane generator of the manifold $X, \operatorname{dim} L=$ $l=n-r ;$ let $T_{L}, \operatorname{dim} T_{L}=n$, be the tangent subspace to $X$ along the generator $L$, and let $M$ be a base manifold for $X, \operatorname{dim} M=r$. Denote by $\theta^{p}, p=l+1, \ldots, n$, basis forms on the variety $M$. These forms satisfy the structure equations

$$
d \theta^{p}=\theta^{q} \wedge \theta_{q}^{p}, \quad p, q=l+1, \ldots, n,
$$

of the variety $M$. Here $\theta_{q}^{p}$ are 1 -forms defining transformations of first-order frames on $M$.

For a point $x \in L$, we have $d x \in T_{L}$. With $X$, we associate a bundle of projective frames $\left\{A_{i}, A_{p}, A_{\alpha}\right\}$ such that $A_{i} \in L, i=0,1, \ldots, l ; A_{p} \in T_{L}, p=l+1, \ldots, n$. Then

$$
\left\{\begin{array}{l}
d A_{i}=\omega_{i}^{j} A_{j}+\omega_{i}^{p} A_{p}, \\
d A_{p}=\omega_{p}^{i} A_{i}+\omega_{p}^{q} A_{q}+\omega_{p}^{\alpha} A_{\alpha}, \alpha=n+1, \ldots, N .
\end{array}\right.
$$

It follows from the first equation of (6) that

$$
\omega_{i}^{\alpha}=0 .
$$

Since for $\theta^{p}=0$ the subspaces $L$ and $T_{L}$ are fixed, we have

$$
\omega_{i}^{p}=c_{q i}^{p} \theta^{q}, \quad \omega_{p}^{\alpha}=b_{q p}^{\alpha} \theta^{q} .
$$

Since the manifold of leaves $L \subset X$ depends on $r$ essential parameters, the rank of the system of 1 -forms $\omega_{i}^{p}$ is equal to $r$, rank $\left(\omega_{i}^{p}\right)=r$. Similarly, we have $\operatorname{rank}\left(\omega_{p}^{\alpha}\right)=r$.

Denote by $C_{i}$ and $B^{\alpha}$ the $r \times r$ matrices occurring in equations (8):

$$
C_{i}=\left(c_{q i}^{p}\right), \quad B^{\alpha}=\left(b_{q p}^{\alpha}\right) .
$$

These matrices are defined in a second-order neighborhood of the submanifold $X$.

Exterior differentiation of equations (7) by means of structure equations (4) leads to the exterior quadratic equations

$$
\omega_{p}^{\alpha} \wedge \omega_{i}^{p}=0
$$

Substituting expansions (8) into the last equations, we find that

$$
b_{q s}^{\alpha} c_{p i}^{s}=b_{p s}^{\alpha} c_{q i}^{s} \text {. }
$$

Equations (7), (8), and (9) are called the basic equations in the theory of tangentially degenerate submanifolds.

Relations (9) can be written in the matrix form

$$
\left(B^{\alpha} C_{i}\right)^{T}=\left(B^{\alpha} C_{i}\right)
$$

i.e., the matrices

$$
H_{i}^{\alpha}=B^{\alpha} C_{i}=\left(b_{q s}^{\alpha} c_{p i}^{s}\right)
$$

are symmetric.

Let $x=x^{i} A_{i}$ be an arbitrary point of a leaf $L$. For such a point we have

$$
d x=\left(d x^{i}+x^{j} \omega_{j}^{i}\right) A_{i}+x^{i} \omega_{i}^{p} A_{p}
$$

It follows that

$$
d x \equiv\left(A_{p} c_{q i}^{p} x^{i}\right) \theta^{q} \quad(\bmod L)
$$


The tangent subspace $T_{x}$ to the manifold $X$ at a point $x$ is defined by the points $A_{i}$ and

$$
\widetilde{A}_{q}(x)=A_{p} c_{q i}^{p} x^{i},
$$

and therefore $T_{x} \subset T_{L}$.

A point $x$ is a regular point of a leaf $L$ if $T_{x}=T_{L}$. The regular points are determined by the condition

$$
J(x)=\operatorname{det}\left(c_{p i}^{q} x^{i}\right) \neq 0 .
$$

If $J(x)=0$ at a point $x$, then $T_{x}$ is a proper subspace of $T_{L}$, and a point $x$ is said to be a singular point of a leaf $L$.

The determinant (10) is the Jacobian of the map $f: P^{l} \times M^{r} \rightarrow P^{N}$. The singular points of a leaf $L$ are determined by the condition $J(x)=0$. In a leaf $L$, they form an algebraic submanifold of dimension $l-1$ and degree $r$. This hypersurface (in $L$ ) is called the focus hypersurface and is denoted by $F_{L}$. By (10), the equations $J(x)=0$ of the focus hypersurface in the plane generator $L$ of the manifold $X$ can be written as

$$
\operatorname{det}\left(c_{p i}^{q} x^{i}\right)=0 .
$$

For the point $x=x^{i} A_{i} \in L$, by (6) we have

$$
d^{2} x \equiv A_{\alpha} b_{q s}^{\alpha} c_{p i}^{s} x^{i} \theta^{p} \theta^{q} \quad\left(\bmod T_{L}\right) .
$$

Thus, the points

$$
A_{p q}=A_{\alpha} b_{q s}^{\alpha} c_{p i}^{s} x^{i}, \quad A_{p q}=A_{q p},
$$

together with the points $A_{i}$ and $A_{p}$ define the osculating subspace $T_{x}^{2}(X)$. Its dimension is

$$
\operatorname{dim} T_{x}^{2}(X)=n+m,
$$

where $m$ is the number of linearly independent points among the points $A_{p q}, m \leq$ $\min \left\{\frac{r(r+1)}{2}, N-n\right\}$. But since at a regular point $x \in L$ condition (10) holds, the number $m$ is the number of linearly independent points among the points

$$
\widetilde{A}_{p q}=A_{\alpha} b_{p q}^{\alpha}
$$

Hence, the osculating subspace $T_{L}^{2}(X)$ is the same for all regular points $x \in L$, and we denote it by $T_{L}^{2}(X)$.

We further assume that the point $A_{0}$ is a regular point of the generator $L$. Then equation (10) implies that $\operatorname{det}\left(c_{q 0}^{p}\right) \neq 0$, and the 1 -forms $\omega_{o}^{p}=c_{q 0}^{p} \theta^{q}$ are linearly independent. Thus, they can be taken as basis forms of the manifold $M^{r}$, i.e., we can set $\omega_{0}^{p}=\theta^{p}$. Then the matrix $C_{0}=\left(c_{q 0}^{p}\right)$ becomes the identity matrix, $c_{q 0}^{p}=\delta_{q}^{p}$. This and equations (9) imply that

$$
b_{p q}^{\alpha}=b_{q p}^{\alpha},
$$

i.e., the matrices $B^{\alpha}$ become symmetric.

The number $m$ coincides with the number of linearly independent matrices $B^{\alpha}=$ $\left(b_{p q}^{\alpha}\right)$ and with the number of linearly independent second fundamental forms

$$
\phi^{\alpha}=b_{p q}^{\alpha} \omega_{0}^{p} \omega_{0}^{q} .
$$

of the submanifold $X$ at its regular point $A_{0}$.

4. Decomposition of the focus hypersurface $\boldsymbol{F}_{\boldsymbol{L}}$. We prove now the following lemma; 
Lemma 1. Suppose that $m \geq 2$, and in the pencil of the second fundamental forms $\xi_{\alpha} \phi^{\alpha}$ of $X$, there are two forms $\phi^{\prime}$ and $\phi^{\prime \prime}$ defining a regular pencil, and all eigenvalues $\lambda_{p}, p=l+1, \ldots, n$, of this pencil defined by the characteristic equation

$$
\operatorname{det}\left(b_{p q}^{\prime \prime}+\lambda b_{p q}^{\prime}\right)=0
$$

are distinct. Then all the matrices $C_{i}=\left(c_{q i}^{p}\right)$ can be diagonalized simultaneously with the matrices $B^{\prime}=\left(b_{p q}^{\prime}\right)$ and $B^{\prime \prime}=\left(b_{p q}^{\prime \prime}\right)$.

Proof. In fact, by the lemma condition, the matrices $B^{\prime}$ and $B^{\prime \prime}$ can be simultaneously diagonalized:

$$
B^{\prime}=\left(b_{p p}^{\prime}\right), \quad B^{\prime \prime}=\left(b_{p p}^{\prime \prime}\right) .
$$

Since all roots of equation (12) are distinct, then

$$
\frac{b_{p p}^{\prime}}{b_{p p}^{\prime \prime}} \neq \frac{b_{q q}^{\prime}}{b_{q q}^{\prime \prime}}, \quad p \neq q .
$$

Consider now equations (9). By (13), equations (9) take the form

$$
b_{q q}^{\prime} c_{p a}^{q}=b_{p p}^{\prime} c_{q a}^{p}, \quad b_{q q}^{\prime \prime} c_{p a}^{q}=b_{p p}^{\prime \prime} c_{q a}^{p},
$$

where $a=1, \ldots, l$. But by the inequality (14), equations (15) imply that

$$
c_{q a}^{p}=0, \quad p \neq q .
$$

Since in addition, we have $C_{0}=\left(\delta_{q}^{p}\right)$, each of the matrices $C_{i}$ has a diagonal form.

Lemma 1 implies the following corollary.

Corollary. Under the conditions of Lemma 1, the focus hypersurface $F_{L}$ of its generator $L$ decomposes into $r$ hyperplanes defined by the equations

$$
x^{0}+c_{p a}^{p} x^{a}=0, a=1, \ldots, l,
$$

where there is no summation over $p$.

In fact, by Lemma 1 , the matrices $C_{0}$ and $C_{a}$ can be simultaneously diagonalized, and $C_{0}=\left(\delta_{q}^{p}\right)$. As a result, equation (11) of the focus hypersurface $F_{L}$ takes the form

$$
\prod_{p=l+1}^{n}\left(x^{0}+c_{p i}^{p} x^{i}\right)=0 .
$$

Therefore, the hypersurface $F_{L}$ decomposes into $r$ hyperplanes (17).

We can reformulate Lemma 1 in geometric terms by using the notion of the focus hypercone $\Phi_{L}$ formed by singular tangent hyperplanes of the submanifold $X$ containing the tangent subspace $T_{L}$. The hypercone $\Phi_{L}$ is determined by the equations

$$
\operatorname{det}\left(\xi_{\alpha} b_{p q}^{\alpha}\right)=0
$$

(see [2], p. 119, or [3]). The new formulation of Lemma 1 is:

If $m \geq 2$, and the focus hypercone $\Phi_{L}$ does not have multiple components, then the focus hypersurface $F_{L}$ of its generator $L$ decomposes into $r$ hyperplanes $F_{p}$ lying in $L$.

5. Sufficient conditions for $\boldsymbol{X}$ to be a cone. Lemma 1 allows us to prove the following lemma giving sufficient conditions for a submanifold $X$ with a degenerate Gauss map to be a cone.

Lemma 2. Suppose that a tangentially degenerate submanifold $X$ satisfies the following conditions: 
(i) The conditions of Lemma 1.

(ii) $r \geq 2$.

(iii) All hyperplanes $F_{p}$, into which by Lemma 1 the focus hypersurface $F_{L}$ decomposes, coincide.

Then the submanifold $X$ is a cone with $(l-1)$-dimensional vertex and l-dimensional plane generators.

Proof. In fact, if all hyperplanes $F_{p}$ defined by equations (17) coincide, then by placing the points $A_{b}, b=1, \ldots, l$, of our moving frame into this hyperplane, we reduce the equation of this hyperplane to the form $x^{0}=0$. As a result, all the coefficients $c_{p i}^{p}$ vanish. Since we also have conditions (16), it follows that

$$
c_{q a}^{p}=0, a=1, \ldots, l,
$$

for all $p, q=l+1, \ldots, n$. Now equations (8) imply that

$$
\omega_{a}^{p}=0 .
$$

Taking exterior derivatives of equations (19), we obtain

$$
\omega_{a}^{0} \wedge \omega_{0}^{p}=0 .
$$

Since the forms $\omega_{0}^{p}$ are linearly independent (they are basis forms of the variety $M^{r}$ ) and $r \geq 2$, it follows that

$$
\omega_{a}^{0}=0
$$

Equations (18) and (19) imply that

$$
d A_{b}=\omega_{b}^{c} A_{c}
$$

i.e., the $(l-1)$-plane $A_{1} \wedge \ldots \wedge A_{l}$ is constant. Thus, the submanifold $X$ is a cone with $(l-1)$-dimensional vertex.

6. Proof of Theorem 1. We have a submanifold $X^{n}$ of dimension $n$ and rank $r$, rank $X=r$, without singularities in an affine space $A^{N}(\mathbb{C}), N-n \geq 2$. Thus all singular points of $X$ are located at a hyperplane at infinity $P_{\infty}^{N-1} \subset \bar{A}^{N}=$ $A^{N} \cup P_{\infty}^{N-1}$.

Now for $r \geq 2$, we can apply Lemma 2. The cones in $P^{N}(\mathbb{C})$ are projectively equivalent to the cylinders $X^{n} \subset A^{N}(\mathbb{C})$ : a cylinder $X^{n} \subset A^{N}(\mathbb{C})$ is obtained from a cone $\tilde{X}^{n} \subset P^{N}(\mathbb{C})$ by means of a projective transformation sending the cone vertex $F, \operatorname{dim} F=l-1$, into $P_{\infty}^{N-1} \subset A^{N}(\mathbb{C})$. The director variety $Y, \operatorname{dim} Y=$ rank $Y=r$, of the cylinder $X^{n}$ lies in the proper subspace $T$, $\operatorname{dim} T=N-l ; T$ is complementary to $F$. Thus, by Lemma $2, X^{n}$ is an $(n-r)$-cylinder erected over an $r$-dimensional submanifold (a director variety) $Y$ and having $(n-r)$-dimensional plane generators.

\section{REFERENCES}

1. Abe, K., A complex analogue of Hartman-Nirenberg cylinder theorem, J. Diff. Geom., 7 (1972), 453-460. (MR 52 \#4188; Zbl 272.53040.)

2. Akivis, M. A. and V. V. Goldberg, Projective differential geometry of submanifolds, NorthHolland, Amsterdam, 1993, xii+362 pp. (MR 94i:53001; Zbl 865:53013.)

3. Akivis, M. A. and V. V. Goldberg, On the structure of submanifolds with degenerate Gauss maps, Geom. Dedicata 86 (2001) (to appear).

4. Akivis, M. A. and V. V. Goldberg, Local equivalence of Sacksteder and Bourgain hypersurfaces, Hokkaido Math. J. 30 (2001), No. 3 (to appear). 
5. Borisenko, A. A., Extrinsic geometry of strongly parabolic multidimensional submanifolds (Russian), Uspekhi Mat. Nauk 52 (1997), no. 6(318), 3-52; English transl: Russian Math. Surveys 52 (1997), no. 6, 1141-1190. (MR 99f:53053; Zbl 935:53004.)

6. Chern, S. S. and R. K. Lashof, The total curvature of immersed manifolds, Amer. J. Math. 79 (1957), 308-318. (MR 18, \#927a; Zbl 78, p. 139.)

7. Hartman, P. and L. Nirenberg, On spherical image whose Jacobians do not change sign, Amer. J. Math. 81 (1959), 901-920. (MR 23 \#A4106; Zbl 94, p. 163.)

8. Ishikawa, G., Singularities of developable surfaces, In: Singularity theory (Liverpool, 1996), xxii-xxiii, 403-418, London Math. Soc. Lecture Note Ser., 263, Cambridge Univ. Press, Cambridge, 1999. (MR 2000m:58067.)

9. Ishikawa, G., Developable hypersurfaces and homogeneous spaces in a real projective space, Lobachevskii J. Math. 3 (1999), 113-125. (MR 2000m:58090.)

10. Nomizu, K. and Pinkall, U., On the geometry of affine immersions, Math. Z. 195 (1987), 165-178. (MR 88e:53089; Zbl 629:53012.)

11. Nomizu, K. and Sasaki, T., Affine differential geometry, Cambridge Tracts in Mathematics, 111, Cambridge: Cambridge University Press, 1994, xiv+263 pp. (MR 96e:53014; Zbl 834:53002.)

12. Opozda, B., Affine differential geometry of degenerate hypersurfaces, In: Geometry and topology of submanifolds, VIII (Brussels, 1995/Nordfjordeid, 1995), 269-279, World Sci. Publishing, River Edge, NJ, 1996. (MR 98e:53011; Zbl 940:53008.)

13. Opozda, B., A characterization of affine cylinders, Monatsh. Math. 121 (1996), no. 1-2, 113-124. (MR 97d:53032; Zbl 843:53012.)

14. Opozda, B., A new cylinder theorem, Math. Ann. 312 (1998), no. 1, 1-12. (MR 2000j:53074; Zbl 843.53012.)

15. Piontkowski, J., Developable varieties with all singularities at infinity, Preprint, 2001.

16. Pogorelov, A. V., Continuous maps of bounded variation (Russian), Dokl. Akad. Nauk. SSSR (N.S.) 111 (1956), 757-759. (MR 19, \#309a; Zbl 72, p. 398.)

17. Pogorelov, A. V., Extensions of the theorem of Gauss on spherical representation to the case of surfaces of bounded extrinsic curvature (Russian), Dokl. Akad. Nauk. SSSR (N.S.) 111 (1956), 945-947. (MR 19, \#309b; Zbl 70, p. 168.)

18. Sacksteder, R., On hypersurfaces with no negative sectional curvature, Amer. J. Math. 82 (1960), no. 3, 609-630. (MR 22 \#7087; Zbl 194, p. 227.)

19. Sternberg, S., Lectures on differential geometry, Prentice-Hall, Inc., Englewood Cliffs, NJ, 1964, 390 pp. (MR 23 \#1797; Zbl 211, 535); 2d ed., Chelsea Publishing, New York, 1983, xi+442 pp. (MR 88f:58001; Zbl 518:53001.)

20. Stoker, J. J., Developable surfaces in the large, Commun. Pure Appl. Math. 14 (1961), 627635. (MR 25 \#493; Zbl 114:36902.)

21. Stoker, J. J., Differential geometry, Wiley-Interscience, New York, 1969, xxi+404 pp. (MR 39 \#2072; Zbl 182:54601.)

22. Wu, H., Complete developable submanifolds in real and complex Euclidean spaces, Internat. J. Math. 6 (1995), no. 3, 461-489. (MR 96e:53083; Zbl 839:53004.)

Authors' addresses:

\section{A. Akivis}

Department of Mathematics

Jerusalem College of Technology-Mahon Lev

Havaad Haleumi St., P. O. B. 16031

Jerusalem 91160, Israel

E-mail address: akivis@avoda.jct.ac.il

\section{V. Goldberg}

Department of Mathematical Sciences

New Jersey Institute of Technology

University Heights

Newark, N.J. 07102, U.S.A.

E-mail address: vlgold@m.njit.edu 